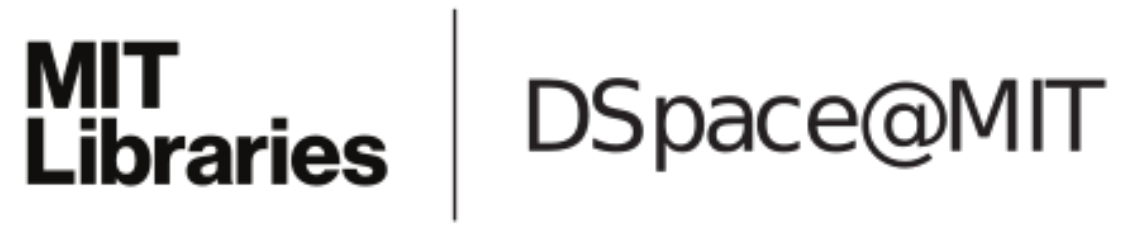

\author{
MIT Open Access Articles
}

Topological pumping over a photonic Fibonacci quasicrystal

The MIT Faculty has made this article openly available. Please share how this access benefits you. Your story matters.

Citation: Verbin, Mor et al. “Topological Pumping over a Photonic Fibonacci Quasicrystal.” Physical Review B 91.6 (2015): 064201-1-064201-6. (C) 2015 American Physical Society

As Published: http://dx.doi.org/10.1103/PhysRevB.91.064201

Publisher: American Physical Society

Persistent URL: http://hdl.handle.net/1721.1/93873

Version: Final published version: final published article, as it appeared in a journal, conference proceedings, or other formally published context

Terms of Use: Article is made available in accordance with the publisher's policy and may be subject to US copyright law. Please refer to the publisher's site for terms of use. 


\title{
Topological pumping over a photonic Fibonacci quasicrystal
}

\author{
Mor Verbin, ${ }^{1}$ Oded Zilberberg, ${ }^{2}$ Yoav Lahini, ${ }^{3}$ Yaacov E. Kraus, ${ }^{1}$ and Yaron Silberberg ${ }^{1}$ \\ ${ }^{1}$ Faculty of Physics, Weizmann Institute of Science, Rehovot 76100, Israel \\ ${ }^{2}$ Institute for Theoretical Physics, ETH Zurich, 8093 Zürich, Switzerland \\ ${ }^{3}$ Department of Physics, Massachusetts Institute of Technology, Cambridge, Massachusetts 02139, USA
}

(Received 8 April 2014; revised manuscript received 18 January 2015; published 4 February 2015)

\begin{abstract}
Quasiperiodic lattices have recently been shown to be a nontrivial topological phase of matter. Charge pumping - one of the hallmarks of topological states of matter-was recently realized for photons in a one-dimensional off-diagonal Harper model implemented in a photonic waveguide array. However, if the relationship between topological pumps and quasiperiodic systems is generic, one might wonder how to observe it in the canonical and most studied quasicrystalline system in one dimension- the Fibonacci chain. This chain is expected to facilitate a similar phenomenon, yet its discrete nature hinders the experimental study of such topological effects. Here, we overcome this obstacle by utilizing the topological equivalence of a family of quasiperiodic models which ranges from the Fibonacci chain to the Harper model. Implemented in photonic waveguide arrays, we observe the topological properties of this family, and perform a topological pumping of photons across a Fibonacci chain.
\end{abstract}

DOI: 10.1103/PhysRevB.91.064201

PACS number(s): 71.23.Ft, 05.30.Rt, 42.82.Cr

\section{INTRODUCTION}

The discovery of topological phases of matter gave birth to an exciting new field of research $[1,2]$. The topological classification of gapped systems such as band insulators and superconductors provides insight into the physical behavior of these systems and predicts novel subgap phenomena [3]. One such phenomenon is the topological pump. Making use of the edge states that appear at the boundary between a topological system and vacuum, a dissipationless current of particles across the sample can be generated through an adiabatic change of the parameters of the system [4-7]. For example, the topologically nontrivial two-dimensional quantum Hall effect (2D QHE) has topologically protected edge states that traverse its energy gaps. Placing it on a cylinder and threading it with an Aharonov-Bohm (AB) flux produces a one-dimensional (1D) quantized charge pump. As the AB flux is continuously increased, an integer number of electrons is transferred across the cylinder for each flux quantum [7-9].

The 2D QHE is deeply related to the 1D Harper model and its off-diagonal variant [10-12]. Whenever the cosine modulation of the model is incommensurate with the underlying lattice, the Harper model describes a quasiperiodic model, i.e., a lattice which is ordered but nonperiodic [13]. In this case, the $\mathrm{AB}$ flux of the 2D QHE becomes equivalent to translations of the 1D Harper model, and boundary states appear in the Harper model as projections of the topologically protected edge states of the 2D QHE. This equivalence suggests that upon a scan of the translational degree of freedom, particles would be pumped from one boundary to the other.

In a recent experiment, the off-diagonal Harper model was implemented in a photonic waveguide array [4]. The quasiperiodic cosine modulation of the model was produced by controlling interwaveguide distances. By adiabatically varying the relative phase between the modulation and the underlying lattice, light was pumped across the sample, revealing its topological nature. Producing a deep connection between topological phases of matter and the seemingly unrelated topic of quasicrystals, this work generated growing interest in the boundary phenomena of quasiperiodic models [14-18]. One development was the discovery of the topological origin of the localized boundary modes of the Fibonacci chain [19] - a binary chain whose lattice spacings are two discrete values that appear interchangeably according to the Fibonacci sequence. Despite the topological properties of this model, when studying its subgap boundary modes it becomes apparent that performing an adiabatic pumping over a Fibonacci chain will be challenging. As adiabatic processes cannot be performed over its discrete potential, it was unclear whether this theoretically proposed process can be realized experimentally.

In this paper, we report a topological pump over a Fibonacci chain implemented in a photonic waveguide array. To achieve this, we harness the recently found topological equivalence between the Fibonacci chain and the Harper model. This equivalence can be accessed through a single deformation parameter whose range spans an extensive family of topologically equivalent quasiperiodic models [19]. We thereby perform a two-parameter topological pumping that includes (i) a deformation of the Fibonacci chain to a smoothened topologically equivalent model, (ii) a scan of the translation parameter, and (iii) a deformation back into a Fibonacci chain. Thus, this paper contains an experimental demonstration of several fundamental concepts, including the deep relationship between topological pumps and quasicrystals, the topological equivalence between different quasiperiodic models, and the connection between their boundary phenomena.

\section{MODELS}

Photonic waveguide arrays [20] serve as a highly versatile and customizable platform to study the properties of topological pumps and of quasicrystals $[4,21]$. In these arrays, evanescent coupling between adjacent waveguides allows photons to hop from one waveguide to another along the propagation axis, denoted by $z$. The resulting dynamics of light propagation is described by a Schrödinger equation with $z$ taking over the role of time, $i \partial_{z} \psi_{n}=H \psi_{n}$, where $\psi_{n}$ is 
(a)

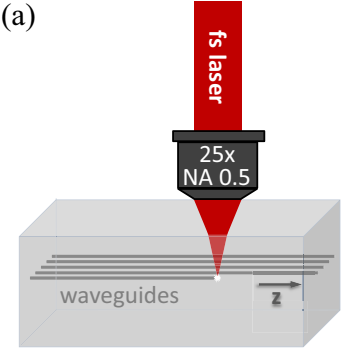

(c)

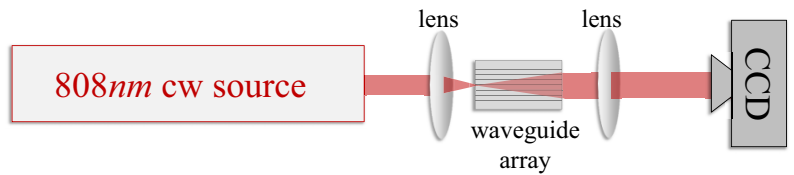

FIG. 1. (Color online) Experimental setup. (a) Pulses from a femtosecond fiber laser (Raydiance Smart Light) with a central wavelength of $1552 \mathrm{~nm}$ are focused inside the glass, resulting in a permanent change to the index of refraction. (b) Microscope image of a waveguide array used in one of the experiments. One waveguide runs from one side of the glass slide to the other and is used for input. The rest of the waveguides start at some distance from input facet of the glass, with the distance determining the propagation length along the array. Inset marker is $10 \mu \mathrm{m}$ long, and the distances between the waveguides range from 8 to $12 \mu \mathrm{m}$. (c) A 808-nm-wavelength beam from a continuous-wave $(\mathrm{cw})$ diode laser is injected into the waveguide array, allowed to propagate along it, and measured at the output using a CCD camera.

the wave function at waveguide number $n . H$ is a general off-diagonal tight-binding Hamiltonian:

$$
H \psi_{n}=t_{n} \psi_{n-1}+t_{n+1} \psi_{n+1},
$$

where $t_{n}$ is the hopping amplitude from waveguide $n$ to waveguide $n-1$.

To introduce quasiperiodicity into the system, the values of $t_{n}$ are modulated according to

$$
t_{n}=t_{0}\left[1+\lambda d_{n}\right],
$$

where $t_{0}$ is the characteristic hopping amplitude of the system, $\lambda \in[0,1)$ is the modulation strength, and $d_{n} \in[-1,1]$ can be any chosen quasiperiodic modulation function.

In the off-diagonal Harper model, the quasiperiodicity enters in the form of a cosine modulation:

$$
d_{n}^{\mathrm{H}}=\cos (2 \pi b n+\phi) .
$$

This model has a long-range order which originates from the sampling of the cosine function, where the modulation frequency $b$ determines the bulk properties of the chain [12]. A quasiperiodic chain is produced whenever the hopping modulation is incommensurate with the underlying lattice (i.e., $b$ is irrational). Correspondingly, the parameter $\phi$ shifts the origin of the modulation. This shift degree of freedom (also known as a phason) corresponds to the AB flux in the 2D QHE and spans a family of models that corresponds to a topological pump [4,8]. Accordingly, topological boundary states appear and disappear as a function of $\phi[21]$.

Comparably, a Fibonacci-like chain is constructed of a sequence of two distinct values which are ordered in a quasiperiodic manner:

$d_{n}^{\mathrm{F}}=2\left(\left\lfloor\frac{\tau}{\tau+1}(n+2)\right\rfloor-\left\lfloor\frac{\tau}{\tau+1}(n+1)\right\rfloor\right)-1= \pm 1$,

where $\lfloor x\rfloor$ is the floor function. This sequence is obtained by applying the "cut-and-project" procedure, i.e., projecting a strip of a square lattice onto the line $y=x / \tau$ [22]. Whenever the slope of the line, $\tau$, is irrational, Eq. (4) becomes quasiperiodic. For example, the case of $\tau=(1+\sqrt{5}) / 2$ is the well-known Fibonacci chain [23]. The absence of a $\phi$-like shift parameter in Eq. (4) is the first obstacle in our attempt to use this model for topological pumping. While such a parameter can be manually inserted into both step functions in the equation, this method will result in discontinuous changes in the model, and is therefore insufficient to achieve adiabatic pumping.

The Harper and Fibonacci models have different physical properties [12,23], and until recently only partial success has been achieved in attempts to combine them under the same general framework [24-26]. A recent paper presented a smooth deformation between the two models, which preserves the topological properties of their energy spectra and enables the definition of a generalized family of topologically equivalent quasiperiodic models [19]:

$$
d_{n}^{\mathrm{S}}(\beta)=\frac{\tanh \left\{\beta\left[\cos \left(2 \pi \bar{b} \cdot \frac{2 n+3}{2}+\phi\right)-\cos (\pi \bar{b})\right]\right\}}{\tanh (\beta)} .
$$

At the limit of $\beta \rightarrow 0, \tanh (\beta x) / \tanh (\beta) \rightarrow x$, yielding the Harper modulation with $\bar{b}=b$, i.e., $d_{n}^{\mathrm{S}} \rightarrow d_{n}^{\mathrm{H}}$, up to a constant shift. At the opposite limit of $\beta \rightarrow \infty, \tanh (\beta x) / \tanh (\beta) \rightarrow$ $\operatorname{sign}(x)$, so for $\bar{b}=\tau /(\tau+1)$ we obtain a Fibonacci chain, i.e., $d_{n}^{\mathrm{S}} \rightarrow d_{n}^{\mathrm{F}}$.

This new family of models can be studied by generating a chain using Eq. (5) and inserting it into the Hamiltonian in Eq. (1) via Eq. (2). The energy spectrum of the resulting Hamiltonian is composed of a set of bands and gaps. The topological index (i.e., the Chern number) of a gap in the energy spectrum which remains open along the deformation is independent of $\beta[19,27]$. This means that different chains with the same irrational modulation frequency $\bar{b}$ are topologically equivalent as long as their gaps remain open as a function of $\beta$. An important outcome of this deformation is that the parameter $\phi$ now gives us a way of incorporating an equivalent of the $\mathrm{AB}$ flux into the Fibonacci chain. Combined with $\beta$, it acts as a controllable knob which allows the experimental observation of boundary states and the generation of a topological pump [19].

\section{EXPERIMENTAL APPARATUS}

To study the topological properties of the different chains produced by Eq. (5), waveguide arrays are fabricated using femtosecond-laser microfabrication technique [28], as illustrated in Fig. 1(a). High-intensity laser pulses are focused inside 75-mm-long bulk glass slides mounted on a computercontrolled stage, producing waveguides which are identical in both refractive index and size. The spatial separation between the waveguides is customized for the desired quasiperiodic 

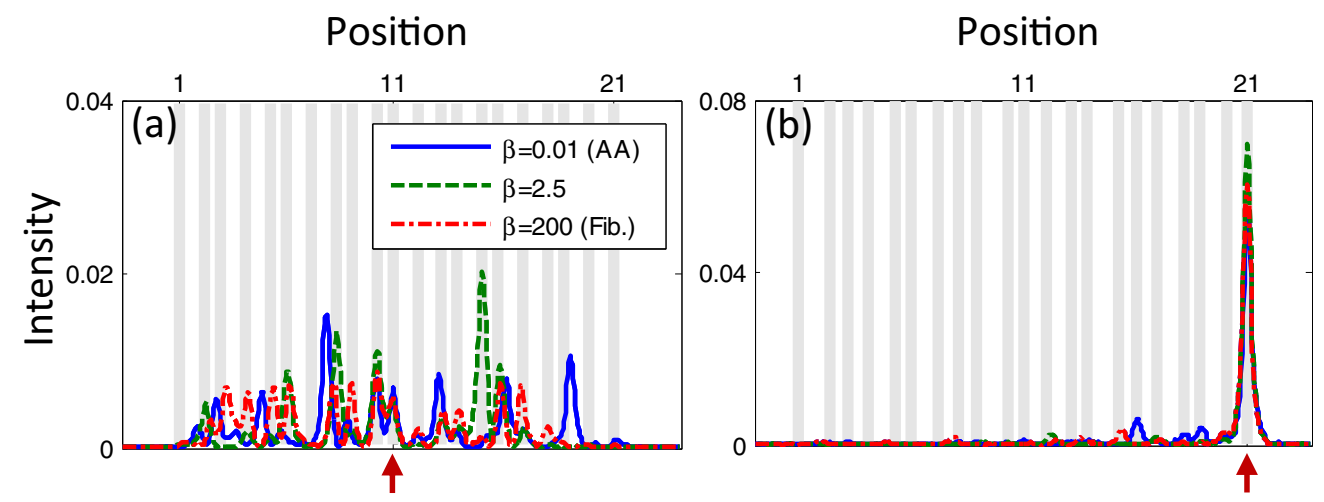

FIG. 2. (Color online) Experimental observation of the right boundary state for 21-waveguide-long arrays with $\beta=0.01$ (a Harper chain), $\beta=2.5$, and $\beta=200$ (a Fibonacci chain), with $\lambda=0.6, \bar{b}=(1+\sqrt{5}) / 2$, and $\phi=0.7 \pi$. Light was initially injected into a single waveguide (red arrows). The measured outgoing intensity is plotted versus the waveguide position. (a) An excitation at the middle of the array (site 11) results in a significant spread of the wave function. (b) Regardless of the value of $\beta$, for an excitation at the rightmost waveguide (site 21) the light remains tightly localized at the boundary, marking the existence of a boundary state.

profile of coupling coefficients using a premeasured distanceto-coupling-coefficient calibration. The resulting structure is visible to an optical microscope, as seen in Fig. 1(b). To study the dynamics of light propagating within the system, a continuous-wave laser beam is injected into one of the waveguides in the array, allowed to propagate along it, and measured at the output, as illustrated in Fig. 1(c). The intensity distribution is then analyzed, producing a normalized one-dimensional distribution, the end result of the system's dynamics.

\section{EXPERIMENTS AND RESULTS}

In order to characterize the family of models presented in Eq. (5) and make use of them to demonstrate an adiabatic pumping over the Fibonacci chain, we have performed three different experiments, detailed here.

\section{A. Observation of edge states}

While the bulk properties of the Harper and Fibonacci models have been studied extensively in the past [12,23], their boundary states have received less attention. Our first experiment is designed to observe boundary states in the generalized model presented in Eq. (5). For this purpose three 21-waveguide-long arrays [29] were fabricated with $\beta=0.01$ (a Harper chain), $\beta=2.5$, and $\beta=200$ (a Fibonacci chain). The resulting experimental observations are depicted in Fig. 2. In all three arrays, light injected into a waveguide in the middle of the array showed significant expansion due to the overlap of the input light with the extended bulk eigenstates of the system. However, when light was injected into the rightmost waveguide, the intensity distribution remained tightly localized at the boundary, revealing the existence of a localized boundary state in all three arrays. This result accentuates the connection between the boundary states of the Fibonacci chain and those of the Harper model, showing their topological origin [30-33].

\section{B. Edge state as a function of $\phi$}

In a second set of experiments, we studied the effect of the parameter $\phi$. In the Harper model $(\beta \rightarrow 0), \phi$ shifts

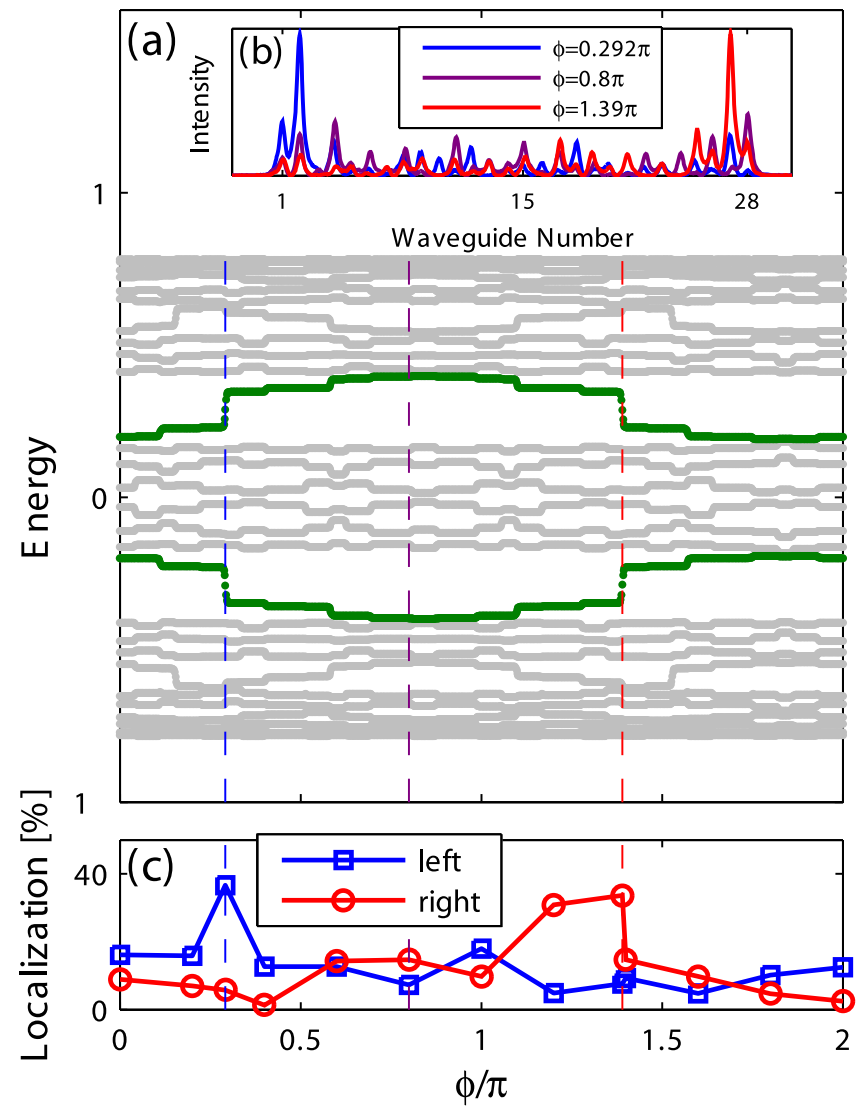

FIG. 3. (Color online) Spectrum and wave-function localization vs $\phi$. (a) Energy spectrum of a 28-site-long Fibonacci chain $(\beta=100)$ vs $\phi$, with $\lambda=0.25$ and $\bar{b}=(1+\sqrt{5}) / 2$. The two boundary states that traverse the largest energy gaps as a function of $\phi$ are marked in green. Blue, purple, and red dashed lines mark three values of $\phi$ $(0.292 \pi, 0.8 \pi$, and $1.39 \pi$, correspondingly) for which experimental results are presented in the inset. (b) Output intensity for light inserted into the (blue) leftmost waveguide, exciting the left-hand-side boundary state; (purple) middle waveguide, when the state is found within the energy band; and (red) rightmost waveguide, exciting the right-hand-side boundary state. (c) The amount of light remaining at the two outermost waveguides vs $\phi$ on the (blue) left-hand and (red) right-hand boundaries. 

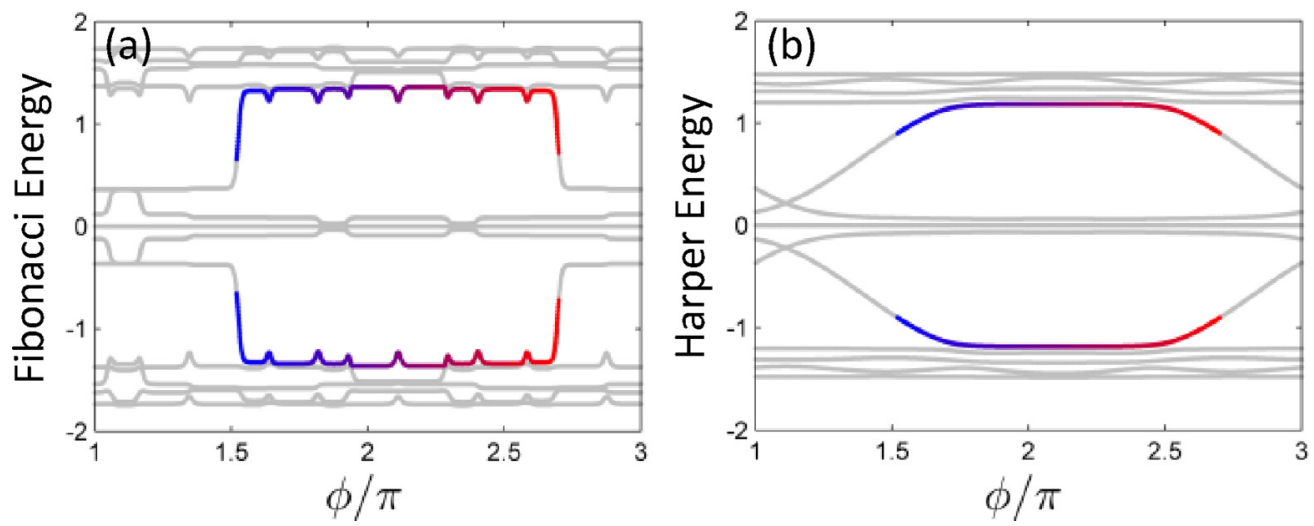

FIG. 4. (Color online) Comparison of spectra. Energy spectra as a function of $\phi$, of a 13-waveguide-long (a) Fibonacci chain $(\beta=200)$ and (b) Harper chain $(\beta=0.01)$ with $\lambda=0.6$.

the location of the boundary state from the right to the left boundary [4]. To study the other limit, at $\beta=100$, thirteen 28-waveguide-long arrays [29] have been fabricated with different values of $\phi$, between 0 and $2 \pi$. Figure 3(a) depicts the numerically obtained energy spectrum of the Hamiltonian of this system as a function of $\phi$. The spectrum is broken into a set of bands and gaps which remains mostly unchanged, but includes two states that counter-traverse the largest energy gaps as a function of $\phi$. When found within the gaps, these states are localized at either the left or right boundary of the system. Light inserted into the corresponding boundaries excites these boundary states and remains there. When these eigenstates are located within the energy band, they behave as bulk states. Accordingly, inserted light spreads across the array, as seen in Fig. 3(b). The amount of light which remained at the two outermost waveguides (closest to the injection sites) as a function of $\phi$ is presented in Fig. 3(c). The two observed peaks correspond to the values of $\phi$ for which boundary states exist on the same side of the array where light was injected. These results show that the dependence of the Fibonacci chain on the parameter $\phi$ is similar to that of the Harper model. It should therefore allow us to perform a topological pumping of photons from one side of a Fibonacci chain to the other.

\section{Adiabatic pumping}

Ideally, pumping could be done in the Fibonacci chain by adiabatically scanning $\phi$ to allow light to follow the localized state, found in the previous experiment, from one side of the array to the other [4]. However, a comparison of the energy spectra of the Fibonacci and Harper models [see Figs. 4(a) and 4(b), respectively], reveals an experimental obstacle. As $\beta$ increases, the region in which the boundary states traverse the gaps becomes shorter, and as we approach the Fibonacci chain they become infinitesimally small [34]. This sharp traversal hinders any adiabatic processes, as these require an infinite propagation length to adiabatically follow the localized state from the energy gap to the energy band. Notwithstanding, this problem can be circumvented by the topological equivalence maintained along the deformation in Eq. (5). Since the topological class of the largest energy

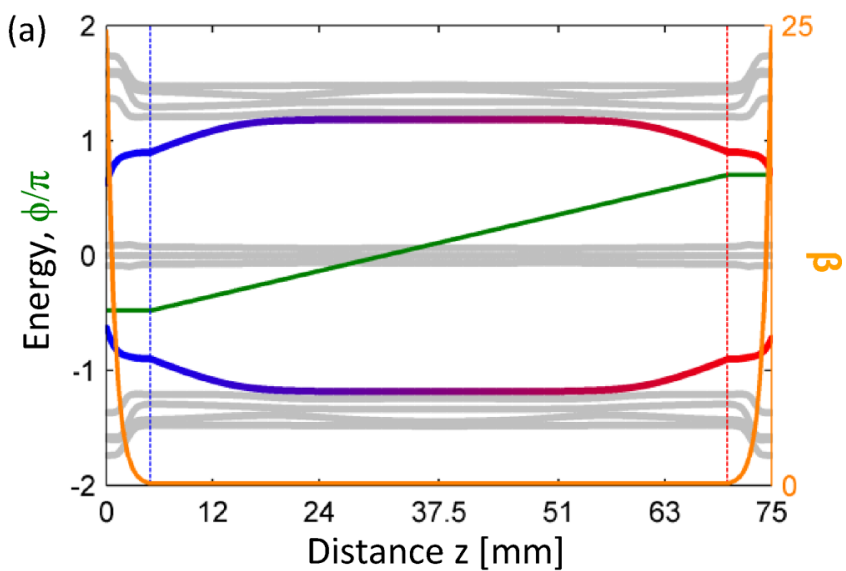

(b)

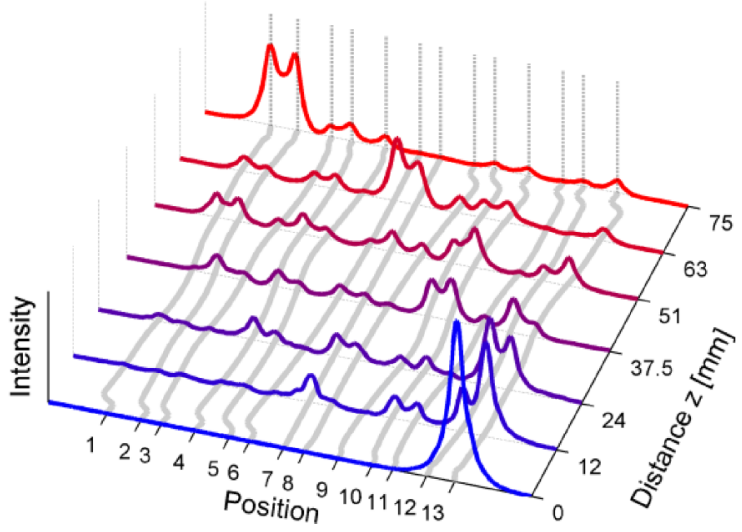

FIG. 5. (Color online) Two-parameter topological pumping. (a) Energy spectrum of a 13-waveguide-long array as a function of the propagation axis $z$, with scanned parameters $\phi$ (green) and $\beta$ (orange). At $z=0$ the array starts as a Fibonacci chain $(\beta=25)$. An adiabatic process lowers $\beta$ to 0.01 , resulting in a Harper chain at $z=5 \mathrm{~mm}$. $\phi$ is then scanned from $-0.48 \pi$ to $0.7 \pi$ to pump light across the structure. Finally, $\beta$ is increased back to 25 between $z=70 \mathrm{~mm}$ and $z=75 \mathrm{~mm}$, ending with a Fibonacci chain. (b) Experimental results of topological pumping. The measured intensity distributions as a function of the position are presented at different stages of the adiabatic evolution, i.e., different propagation distances. Light was injected into the rightmost waveguide (site 13), pumped across the array from right to left, and ended up localized at the two leftmost waveguides (sites 1 and 2). 
gaps of the chain is independent of $\beta$, we can start with an adiabatic deformation of the Fibonacci chain into a Harper model by decreasing $\beta$, then adiabatically $\operatorname{scan} \phi$ for the pumping process, and end with another adiabatic deformation back into a Fibonacci chain.

The structure used for the two-parameter topological pumping is described in Fig. 5(a). Values of $\beta$ and $\phi$ are changed at different stages along the propagation axis, and the resulting energy spectrum varies slowly enough to let light follow the edge state from one side of the array to the other. The transition from a Fibonacci to a Harper chain (instigated by a change of the parameter $\beta$ ) remains adiabatic as long as the localized states are far from the energy bands, and therefore can be done quickly, leaving enough propagation length for an adiabatic pumping along the Harper chain and a transition back to a Fibonacci chain.

This structure was implemented in seven 13-waveguidelong [29] arrays of increasing propagation length, with the same scans of $\beta$ and $\phi$. This allowed for an observation of different steps along the adiabatic propagation, where the dynamics starts at a controlled distance along the single excited waveguide, and stops at the output facet. The experimental results are shown in Fig. 5(b). The snapshots along the propagation axis show the initially excited localized state leaving the right boundary of the Fibonacci chain, expanding along the waveguide array as it propagates, and relocalizing at the other side. This flow of light across the array is a genuine topological pumping of photons.

\section{SUMMARY}

In this paper we have experimentally studied and verified the topological properties of a generalized family of quasiperiodic models which ranges from the Harper model to the Fibonacci chain. All the members of this family have boundary states that appear and disappear as a function of the translation degree of freedom, $\phi$. Members of this family which can be deformed into each other while keeping the main energy gaps open are topologically equivalent. Using this equivalence, we circumvent the obstacles which arise when trying to perform a topological pumping across a Fibonacci chain. Thus, we harness the mathematical notion of topological equivalence to solve an experimental problem. Additionally, the realization of a topological pump across a Fibonacci chain serves as an experimental proof of the topological equivalence between a manifold of quasiperiodic models.

\section{ACKNOWLEDGMENTS}

We thank the Minerva Foundation, US Army RFECAtlantic, the Swiss National Science Foundation, the Pappalardo Fellowship in Physics, and the I-CORE program of the ISF for financial support.
[1] C. L. Kane and E. J. Mele, $Z_{2}$ Topological order and the quantum spin Hall effect, Phys. Rev. Lett. 95, 146802 (2005).

[2] M. König, S. Wiedmann, C. Brüne, A. Roth, H. Buhmann, L. W. Molenkamp, X.-L. Qi, and S.-C. Zhang, Quantum spin Hall insulator state in HgTe quantum wells, Science 318, 766 (2007).

[3] M. Z. Hasan and C. L. Kane, Colloquium: Topological insulators, Rev. Mod. Phys. 82, 3045 (2010); X.-L. Qi and S.-C. Zhang, Topological insulators and superconductors, ibid. 83, 1057 (2011).

[4] Y. E. Kraus, Y. Lahini, Z. Ringel, M. Verbin, and O. Zilberberg, Topological states and adiabatic pumping in quasicrystal, Phys. Rev. Lett. 109, 106402 (2012).

[5] L. Fu and C. L. Kane, Time reversal polarization and a $Z_{2}$ adiabatic spin pump, Phys. Rev. B 74, 195312 (2006).

[6] D. Meidan, T. Micklitz, and P. W. Brouwer, Optimal topological spin pump, Phys. Rev. B 82, 161303 (2010); Topological classification of adiabatic processes, 84, 195410 (2011).

[7] R. B. Laughlin, Quantized Hall conductivity in two dimensions, Phys. Rev. B 23, 5632 (1981).

[8] D. J. Thouless, Quantization of particle transport, Phys. Rev. B 27, 6083 (1983).

[9] D. J. Thouless, M. Kohmoto, M. P. Nightingale, and M. den Nijs, Quantized Hall conductance in a two-dimensional periodic potential, Phys. Rev. Lett. 49, 405 (1982).

[10] P. G. Harper, Single band motion of conduction electrons in a uniform magnetic field, Proc. Phys. Soc., London, Sect. A 68, 874 (1955).

[11] S. Aubry and G. André, Analyticity breaking and Anderson localization in incommensurate lattices, Ann. Isr. Phys. Soc. 3, 133 (1980).
[12] J. H. Han, D. J. Thouless, H. Hiramoto, and M. Kohmoto, Critical and bicritical properties of Harpers equation with next-nearest-neighbor coupling, Phys. Rev. B 50, 11365 (1994).

[13] D. Shechtman, I. Blech, D. Gratias, and J. W. Cahn, Metallic phase with long-range orientational order and no translational symmetry, Phys. Rev. Lett. 53, 1951 (1984); D. Levine and P. J. Steinhardt, Quasicrystals: A new class of ordered structures, ibid. 53, 2477 (1984).

[14] L.-J. Lang, X. Cai, and S. Chen, Edge states and topological phases in one-dimensional optical superlattices, Phys. Rev. Lett. 108, 220401 (2012).

[15] F. Mei, S.-L. Zhu, Z.-M. Zhang, C. H. Oh, and N. Goldman, Simulating $Z_{2}$ topological insulators with cold atoms in a onedimensional optical lattice, Phys. Rev. A 85, 013638 (2012).

[16] S. Ganeshan, K. Sun, and S. Das Sarma, Topological zero-energy modes in gapless commensurate Aubry-André-Harper models, Phys. Rev. Lett. 110, 180403 (2013).

[17] I. I. Satija and G. G. Naumis, Chern and Majorana modes of quasiperiodic systems, Phys. Rev. B 88, 054204 (2013).

[18] O. Viyuela, A. Rivas, and M. A. Martin-Delgado, Thermal instability of protected end states in a one-dimensional topological insulator, Phys. Rev. B 86, 155140 (2012).

[19] Y. E. Kraus and O. Zilberberg, Topological equivalence between the Fibonacci quasicrystal and the Harper model, Phys. Rev. Lett. 109, 116404 (2012).

[20] F. Lederer, G. I. Stegeman, D. N. Christodoulides, G. Assanto, M. Segev, and Y. Silberberg, Discrete solitons in optics, Phys. Rep. 463, 1 (2008).

[21] M. Verbin, O. Zilberberg, Y. E. Kraus, Y. Lahini, and Y. Silberberg, Observation of topological phase transitions in photonic quasicrystals, Phys. Rev. Lett. 110, 076403 (2013). 
[22] M. Senechal, Quasicrystals and Geometry (Cambridge University Press, Cambridge, 1996).

[23] M. Kohmoto, L. P. Kadanoff, and C. Tang, Localization problem in one dimension: Mapping and escape, Phys. Rev. Lett. 50, 1870 (1983); S. Ostlund, R. Pandit, D. Rand, H. J. Schellnhuber, and E. D. Siggia, One-dimensional Schrödinger equation with an almost periodic potential, ibid. 50, 1873 (1983).

[24] H. Hiramoto and M. Kohmoto, Electronic spectral and wavefunction properties of one-dimensional quasiperiodic systems: A scaling approach, Int. J. Mod. Phys. B 6, 281 (1992).

[25] H. Hiramoto and M. Kohmoto, New localization in a quasiperiodic system, Phys. Rev. Lett. 62, 2714 (1989).

[26] G. G. Naumis and F. Lopez-Rodriguez, The electronic spectrum of a quasiperiodic potential: From the Hofstadter butterfly to the Fibonacci chain, Physica B 403, 1755 (2008).

[27] I. Dana, Topologically universal spectral hierarchies of quasiperiodic systems, Phys. Rev. B 89, 205111 (2014).

[28] A. Szameit, D. Blömer, J. Burghoff, T. Schreiber, T. Pertsch, S. Nolte, A. Tünnermann, and F. Lederer, Discrete nonlinear localization in femtosecond laser written waveguides in fused silica, Opt. Express 13, 10552 (2005).
[29] The sizes of the waveguide arrays are limited by our experimental setup. However, simulations guarantee that this behavior persists as the size of the system increases. These simulations do not provide additional information and are therefore not included in this paper.

[30] E. S. Zijlstra, A. Fasolino, and T. Janssen, Existence and localization of surface states on Fibonacci quasicrystals: A tight-binding study, Phys. Rev. B 59, 302 (1999).

[31] Y. El Hassouani, H. Aynaou, E. H. El Boudouti, B. DjafariRouhani, A. Akjouj, and V. R. Velasco, Surface electromagnetic waves in Fibonacci superlattices: Theoretical and experimental results, Phys. Rev. B 74, 035314 (2006).

[32] X.-N. Pang, J.-W. Dong, and H.-Z. Wang, Photonic localization of interface modes at the boundary between metal and Fibonacci quasiperiodic structure, J. Opt. Soc. Am. B 27, 2009 (2010).

[33] A. J. Martínez and M. I. Molina, Surface solitons in quasiperiodic nonlinear photonic lattices, Phys. Rev. A 85, 013807 (2012).

[34] This transition is immediate for a Fibonacci chain at $\beta \rightarrow \infty$, where no gap-traversing topological boundary state exists. 